\title{
Acciones públicas de sensibilización de género. El esfuerzo de la administración regional y local en comunicación publicitaria (1999-2007)
}

\author{
Natalia PAPÍ-GÁLVEZ ${ }^{1}$ \\ Belén CAMBRONERO-SAIZ ${ }^{2}$
}

Universidad de Alicante

\begin{abstract}
RESUMEN:
El artículo analiza las iniciativas de comunicación relacionadas con la igualdad de oportunidades entre mujeres y hombres. El objetivo es analizar con enfoque de género las acciones realizadas por parte de las instituciones públicas regionales y locales para sensibilizar y promover la conciliación entre la vida familiar y laboral de seis comunidades autónomas (Andalucía, Cataluña, Comunidad Valenciana, Galicia, Madrid y País Vasco) y de sus capitales, desde 1999 (cuando se aprueba la ley de conciliación) hasta 2007 (se aprueba la ley de igualdad). La información sobre publicidad convencional fue proporcionada por Infoadex. Para la recogida de otro tipo de iniciativas a través de medios menos convencionales se acudió a cuatro fuentes institucionales: 1) Ayuntamientos de las capitales, como fuente estrictamente local, 2) Diputaciones de la capital de la Comunidad Autónoma, como fuente provincial, 3) Direcciones Generales de la Mujer o instituciones análogas como fuente de información a nivel regional o autonómico y, por último, 4) otras concejalías vinculadas al tema de la corresponsabilidad, cuyo radio de acción también es autonómico. Los resultados, aunque con diferencias entre regiones, reflejan carencias en la cobertura de las acciones que llevan a cuestionar la falta de estrategia política en términos de comunicación, pese a la introducción sistemática de estos objetivos en la agenda internacional (ONU y UE) y del gobierno español. En consecuencia, las campañas publicitarias e iniciativas recogidas contribuyen a la visibilización del problema y al empoderamiento, pero no tanto al objetivo de la paridad.
\end{abstract}

PALABRAS CLAVE: Concienciación; igualdad de género; publicidad institucional; políticas públicas; conciliación de vida familiar y laboral.

TITLE: Public actions of gender awareness. The efforts of regional and local government in advertising communication (1999-2007)

\begin{abstract}
:
The article analyses the communication initiatives related to equal opportunities between women and men. The aim is to To use a gender approach to analyze the actions taken by the regional and local government institutions to promote the work-family reconciliation in six areas (Andalusia, Catalonia, the Region of Valencia, Galicia, Madrid and the Basque Country) from 1999 (when the law of work-family reconciliation is passed) to 2007 (the law of effective equality is passed). The information concerning conventional adver-
\end{abstract}

1 Profesora del departamento de Comunicación y Psicología Social en la licenciatura de Publicidad y Relaciones Públicas (Introducción a la investigación de medios publicitarios). Universidad de Alicante. Email: Natalia.p@ua.es. Trabajo inserto en el proyecto 125/05, plan nacional I+D+i, subvencionado por el Instituto de la Mujer (2006-2009). Grupo de Investigación Estudios de Género en Comunicación, Publicidad y Políticas para la Igualdad.

Personal Investigador en Formación. Universidad de Alicante. Email: belen.cambronero@ua.es. 
tising was provided by Infoadex. To get information of less conventional media, we use four types of institutional resources:1) city councils of the capitals of the six regions, as a strictly local source, 2) provincial councils of the capitals, as a provincial source, 3 ) regional directorates for women or similar institutions as a source of information on a regional level, and finally, 4) other council departments linked to the theme of co-responsibility, with a regional scope of action. Although the results show differences between regions, they highlight deficiencies in terms of parity and mainstreaming. The limitations detected in the number and type of actions taken, therefore, prompt us to question the existence of a medium to long-term political strategy as regards communication and education, despite the systematic introduction of these objectives to the international agenda and that of the Spanish government. The advertising campaigns and collected initiatives contribute to enhancing the visibility of the problem, and the actions taken fundamentally contribute to empowerment, but do not work towards the objective of parity.

KEY WORDS: Awareness; gender equality; institutional advertising; public policy; work-family reconciliation

\section{Introducción}

\subsection{La sensibilización de género como factor de cambio social}

La sensibilización es una toma de conciencia. En materia de igualdad de género, emerge como un objetivo estratégico recogido en la emblemática Declaración de Beijing de 1995. Constituye una de las tres acciones apoyadas por el V Programa para la igualdad de oportunidades ${ }^{3}$ y una de las cuatro acciones del Plan de trabajo de la Unión Europea ${ }^{4}$. Según estos documentos, sensibilizar en igualdad de género es tratar de que la sociedad perciba y asuma el valor de los temas relacionados con la igualdad de oportunidades entre mujeres y hombres. Por tanto, hablar de sensibilización es aceptar la posibilidad de cambio.

Desde el proyecto político de la igualdad de oportunidades, esta consideración de la sensibilización (como factor de cambio) implicaría una estrategia a largo plazo de educación en valores de igualdad y de respeto a la diferencia (que serían las sendas propuestas del feminismo de la igualdad y de la diferencia), en la que tanto los gobiernos como los medios de comunicación tienen un papel crucial ${ }^{5}$.

La sensibilización es, como consecuencia, un objetivo de carácter político que implica una estrategia de naturaleza comunicativa, lo que le lleva a ser objeto de comunicación y/o publicidad institucional. De hecho, la sensibilización está implí-

3 European Commission (2000): Council Decision 2001/51/EC of 20 December 2000 establishing a Programme relating to the Community framework strategy on gender equality (2001-2005), Brussels, Directorate-General for Employment, Social Affairs and Equal Opportunities.

4 EUROPEAN COMMISSION (2006): Roadmap for equality between women and men 2006-2010, Communication from the Commission to the Council, the European Parliament, Brussels, European Economic and Social Committee and the Committee of the Regions.

5 Ministerio De IGUALDAD (2008): Plan Estratégico de Igualdad de Oportunidades entre Mujeres y Hombres (2008-2011), Madrid, Ministerio de Igualdad. 
cita en la ley $29 / 2005^{6}$ cuando expone como una finalidad de la comunicación institucional «promover la difusión y conocimiento de los valores y principios constitucionales» ${ }^{7}$, entre ellos la no discriminación por razón de sexo. Igualmente, la Constitución Española contempla la posibilidad de que las comunidades autónomas adquieran ciertas competencias en ese terreno que son gestionadas por la administración regional. Por lo que algunas comunidades autónomas disponen de leyes análogas que también incluyen la sensibilización. Todas ellas, además, tienen planes de igualdad de oportunidades que apuntan la necesidad de realizar campañas de sensibilización.

Próximos a este planteamiento, algunos estudios analizan el género en las campañas de sensibilización en otros países, como el modelo de padre transmitido por las campañas de comunicación realizadas por el gobierno sueco sobre el permiso de paternidad ${ }^{8}$. En España se han estudiado las campañas de comunicación institucionales (gubernamentales) cuyo objetivo es la sensibilización para la integración de ciertos grupos sociales como los discapacitados ${ }^{9}$ o los inmigrantes ${ }^{10}$.

De la comunicación y publicidad institucional se destaca no solo su carácter estratégico, sino también su carácter relacional. La comunicación de la institución pública ha sido entendida, entre otras cosas, como «un proceso transaccional» en virtud del sistema expresión-interpretación por el cual «se pretende compartir una realidad cognoscitiva ${ }^{11}$. En esta misma línea, se entiende la publicidad institucional como un proceso de comunicación entre las instituciones públicas y la sociedad civil que no se agota en las estrategias publicitarias, sino que interviene en los procesos de construcción social ${ }^{12}$. Al poner el acento en la comunicación, los autores citados asumen que las administraciones públicas pueden intervenir en las representaciones simbólicas y en la conciencia social, lo que permite tender una pasarela entre la comunicación institucional y los marcos interpretativos de la teoría feminista de la comunicación mediática.

6 España. Jefatura Del Estado (2005): Ley de Publicidad y Comunicación Institucional (Ley 29/2005), BOE 312 (2005/12/30), 42902-42905.

7 Ibidem, 42903.

8 KLINTH, R. (2008): «The Best of Both Worlds? Fatherhood and Gender Equality in Swedish paternity Leave Campaigns, 1976-2006», Fathering, 1, New York, MetaPress, 20-39.

9 DurÁN, R. y GINER, J. I. (1999): «Vivir la discapacidad (campaña de sensibilización y cambio de actitudes sobre la discapacidad en edad escolar)», Revista de Servicios Sociales y Política Social, 48, Madrid, Consejo General de Colegios de Diplomados en Trabajo Social, 75-84.

10 MartíneZ, E; VizcaínO-LaORGA, J.; Gavilán, R. (2008): «Government Advertising: an integrative element. The legal framework in Spain», Revista Latina de Comunicación Social, 63, La Laguna, Laboratorio de Tecnologías de la Información y Nuevos Análisis de Comunicación Social.

11 CAnel, M. J. (2007): Comunicación de las Instituciones Públicas, Madrid, Tecnos, 31.

12 García, M. (2001): Publicidad Institucional: el Estado anunciante, Málaga, Servicio de Publicaciones e Intercambio Científico de la Universidad de Málaga. 


\subsection{El carácter relacional de la comunicación: respuestas de la teoría feminista}

Los Women's Studies recogen las aportaciones de las diferentes teorías de la comunicación social ${ }^{13,14}$ para comprender el papel de los medios en el sistema social y los procesos vinculados a la producción y a la recepción ${ }^{15}$, gestándose un corpus propio: los Feminist Media Studies ${ }^{16}$.

En la década de los 80 , la consideración de los medios de comunicación como mediaciones y el desarrollo de los estudios de recepción abre una línea de investigación con enfoque de género muy prolífera, centrada, fundamentalmente, en el medio televisión y en la publicidad ${ }^{17}$.

La teoría de género se basa en la construcción social del sexo, por lo que las ideas motrices sobre las que pivotan los estudios culturales -tales como ideología, hegemonía, resistencia e identidad ${ }^{18}$ - son rápidamente asimiladas. Otros postulados postestructuralistas influyen en el análisis feminista y de género, como son el concepto de poder de Foucault y/o el de deconstrucción de Derrida.

Los estudios culturales pioneros asumen que los medios de comunicación legitiman la ideología dominante. Así, la teoría feminista de la comunicación analiza las representaciones de género transmitidas por los mass media en tanto contenidos de y para el sistema normativo-cultural de una sociedad (el sistema de género), lo que implica aceptar que los medios tienen un efecto sobre las audiencias.

Sin embargo, bajo la influencia de los estudios de recepción y la aceptación de una cultura popular se llega a considerar que la audiencia selecciona los contenidos y los utiliza de acuerdo con las características que pueden condicionar su interpretación (ej. el sexo y/o la etnia). Esta última consideración aleja los estudios culturales del rol socializador de los medios, determinante y único, y los conduce a analizar los procesos de producción y las condiciones en la recepción de los mensajes. De ahí la posibilidad de asumir que la aplicación de la teoría feminista al análisis de la televisión puede contribuir al proceso de la deconstrucción de la ideología,

13 Wolin, L. (2003): «Gender Issues in Advertising- An Oversight Synthesis of Research: 19702002», Journal of Advertising Research, 1, Cambridge, Cambridge University Press, 111-129.

14 Dow, B. J.; CondiT, C. (2005): «The State of the Art in Feminist Scholarship in Communication», Journal of Communication, 3, Washington: ICA / Blackwell Publishing, 448-478.

15 Revisión de los principales temas tratados y respuestas de la teoría feminista en el capítulo 5, «Los medios de comunicación y el enfoque de género» de PAPí-GÁLVEZ, N (2008): El género entre bastidores. El caso de las periodistas de la Comunidad Valenciana, Alicante, Universidad de Alicante, 129-146.

16 VAn Zoonen, L. (2004): Feminist Media Studies, London, SAGE Publications

17 Mattelart, A.; Mattelart, M. (1998): Theories of Communication: A Short Introduction, Inbunden, Sage Publications.

18 Mattelart, A.; Neveu, E. (2003): Introducción a los estudios culturales, Barcelona, Paidós. 
aspecto por otra parte fundamental para la existencia del proyecto político de los feminismos ${ }^{19,20}$.

Desde esta perspectiva, para generar un cambio en el sistema a través de los medios de comunicación, la intervención debe ser global y sostenida en el tiempo. Se deben modificar los contenidos e incidir en los sistemas de producción. Por lo que, si la sensibilización de género consiste en incorporar a la sociedad y a los ciudadanos valores no sexistas, desde este punto de vista se precisa intervenir en las representaciones de género que reproducen estereotipos sociales y sustituir estos contenidos por otros que ayuden al proyecto de la igualdad.

\subsection{El carácter estratégico: el enfoque del marketing social}

Las acciones de sensibilización en materia de género también se pueden enmarcar en lo que se ha venido llamando marketing social, que utiliza las técnicas propias del marketing orientadas al cambio de ideas (creencias, actitudes o valores) y/o prácticas (actos o conductas): bien para estimular aquellas que se consideran beneficiosas para la sociedad bien para inhibir aquellas que son perjudiciales ${ }^{21}$. Este marketing es realizado por las entidades no empresariales, entre las que destacan las administraciones públicas ${ }^{22}$. La Administración emerge, así, como una de las principales promotoras para llevar a cabo campañas de sensibilización, con el propósito de dar respuesta al compromiso adquirido en el contexto internacional ${ }^{23}$.

El compromiso y/o el proyecto político del marketing social se ajustan a los objetivos que pretende conseguir la organización. La postura de este marketing ante la inmediatez y la naturaleza del cambio social contrastan con la visión de los Feminist Media Studies, pues carece de la visión crítica que impregna la teoría feminista. A cambio, proporciona claves estratégicas y una metodología de trabajo para conseguir, en la medida de lo posible, los objetivos perseguidos por la acción realizada.

En este sentido, Santesmases expone que «para llevar a cabo programas de marketing social que supongan cambios sociales debe delimitarse con precisión el comportamiento social a modificar o reforzar, definir el objetivo a alcanzar, res-

19 Para el propósito de este estudio no se atenderá la tendencia apolítica de los estudios culturales, considerados por muchos autores en términos críticos, justamente por el abandono de los marcos explicativos proporcionados por la sociología y la economía. Ver MATTELART, A.; NeVEU, E. (2003), op.cit.

20 Brown, M. E. (1994): «Feminist Culturalist Televisión Criticism - Culture, Theory and Practice», en Mary Ellen Brown (ed.): Television and Women's Culture. The Politics of the Popular, London, Sage Publications, 11-22.

21 KotLer, P.; RoBerto, E. (1989): Social Marketing: Strategies for Changing Public Behavior, New York, Free Press, 1992, 30.

22 Kotler, P.; LeE, N. (2006): Marketing in the Public Sector: A Roadmap for Improved Performance, Philadelphia, Wharton School Publishing.

23 Instituto DE LA MuJer (1999): Las Conferencias Mundiales de Naciones Unidas sobre las Mujeres, Madrid, Ministerio de Trabajo y Asuntos Sociales. 
ponsabilizar a alguna entidad para dirigir el cambio y utilizar una o más estrategias» (Santesmases, 1999: 918) ${ }^{24}$. Y en este empeño la comunicación publicitaria es fundamental ${ }^{25}$, pues las raíces del marketing social «están en el enfoque informativo, en la forma denominada publicidad social» (Santesmases, 1999: 912) ${ }^{26}$.

Desde esta perspectiva, parece oportuno que el proyecto de la sensibilización incluya la comunicación publicitaria, sin cerrar la estrategia a otras formas no convencionales planteada desde una segmentación apropiada del público ${ }^{27}$.

\subsection{División sexual del trabajo y opinión favorable a los roles tradicionales}

A la luz de los resultados que proporcionan las encuestas, la función sensibilizadora de la comunicación institucional queda plenamente justificada.

La división sexual de trabajo sigue existiendo en España y se refleja en la situación laboral de las mujeres. Ellas están menos empleadas que los hombres y, cuando lo están, se sitúan en mayor número en puestos de media jornada ${ }^{28}$. La familia o las responsabilidades domésticas son los principales motivos que llevan a las mujeres a tener un trabajo de estas características ${ }^{29}$. Además, estudios recientes confirman la división tradicional de tareas entre mujeres y hombres dentro del hogar ${ }^{30,31}$.

En el terreno de la opinión, una parte importante de la población española considera que, en el caso de que alguno de los dos miembros de la pareja tuviera que trabajar menos para ocuparse de las tareas del hogar y del cuidado de los hijos, éste debería ser la mujer ${ }^{32}$.

De estos resultados se extraen, al menos, dos lecturas. Por un lado, los datos manifiestan la insuficiencia de las medidas relacionadas con la igualdad de género, con la corresponsabilidad y con la conciliación de la vida familiar y laboral. Por otro, esta situación justifica la importancia adquirida por la corresponsabilidad en el proyecto político de la igualdad de género en los últimos años en España y, en concreto, de la sensibilización. De hecho, los planes nacionales y autonómicos actualmente en vigor siguen reflejando la necesidad de encontrar soluciones y de

24 SAnTESMASES Mestre, M. (1999): Marketing. Conceptos y estrategias, 4ª Ed., Madrid, Pirámide, 918.

25 Rice, R. E.; ATKIN, C.K. (eds.) (2001): Public Communication Campaigns, California, Sage Publication.

26 SANTESMASEs Mestre, M. (1999), op. cit., 912.

27 PAPí-GÁlVEZ, N.; Orbea MirA, J. (2011): Comunicación institucional eficaz en igualdad de género. Sensibilizar en corresponsabilidad. Claves y recomendaciones, Madrid, Asociación de la Comunicación Publicitaria.

28 INE(2009a): Mujeres y hombres en España 2009, Madrid, INE.

29 INE (2009b): Encuesta de Población Activa, ler trimestre de 2009, Madrid, INE.

30 Cis (2008): Barómetro de Junio, $n^{\circ}$ 2766, Madrid, CIS.

31 CIS (2004): Opiniones y actitudes sobre la familia, $n^{\circ} 2578$, Madrid, CIS.

32 CIS (2008), op. cit. 
realizar campañas y acciones de concienciación con la finalidad de producir algún efecto en esta dirección.

\subsection{La corresponsabilidad como respuesta}

La corresponsabilidad defiende un reparto equilibrado entre sexos de las responsabilidades y decisiones relacionadas tanto con la familia como con el ámbito laboral. En 1975, la I Conferencia Mundial de la Mujer de la ONU recoge la necesidad de este reparto para conseguir la igualdad de género. A partir de este momento, la relación entre el trabajo y la familia será un objetivo prioritario tanto en los programas internacionales como en los planes nacionales y regionales españoles.

La idea de la corresponsabilidad quedará entrelazada con las políticas de conciliación entre la vida familiar y laboral ${ }^{33}$. Sin embargo, con diferencias en su discur$\mathrm{so}^{34}$, en términos generales se observa que estas políticas están más cercanas a las políticas de empleo que a las de igualdad de género en toda Europa, ya que suelen intervenir en el tiempo dedicado al trabajo remunerado ${ }^{35}$, circunstancia que suele afectar más a las mujeres. Las políticas de conciliación (o reconciliación) no necesariamente promueven la equidad de género de los roles familiares, pues, aunque algunas pueden suavizar la presión de la doble jornada, también pueden reforzar el sistema de género (como la media jornada).

Esta consideración queda patente en las políticas (planes y leyes) españolas. Durante siete años, España constituye un marco jurídico en el que se refleja, sobre todo simbólicamente, la preocupación en torno a la conciliación y a su relación con la igualdad de género. Se inicia con la Ley 39/1999 que sólo plantea leves modificaciones en materia de permisos laborales ${ }^{36}$ sin suponer un cambio real hacia la igualdad de oportunidades. De hecho, el análisis textual y de contenido de esta ley en comparación con otras políticas permite observar cómo la conciliación es tratada como una solución a la incompatibilidad de jornadas efectuadas por una persona, normalmente la mujer, alejándose de fomentar la distribución de responsabilidades ${ }^{37}$. Con posterioridad, la Ley 39/2006 trata de proveer de un sistema de ayuda asistencial en casos de dependencia. De alguna forma, beneficiaría a las mujeres en tanto principales cuidadoras, aun-

33 Stratigaki, M. (2004): «The cooptation of Gender Concepts in EU Policies: The Case of Reconciliation of Work and Family», Social Politics, 1, Oxford, Oxford University Press, 30-56.

34 Duncan, S. (2002): «Policy discourses on "Reconciling Work and Life" in the EU», Social Policy $\&$ Society, 1, Cambridge, Cambridge University Press, 305-314.

35 Stratigaki, M. (2004), op. cit,. 30.

36 EsPaña. JeFATURA Del Estado (1999): Ley de la Conciliación de la vida familiar y laboral (Ley 39/1999), BOE 266 (1999/11/06), 38934-38942.

37 PAPí-GÁlveZ, N. (dir.) (2004): Evaluación del impacto de la conciliación entre la vida familiar y profesional para la igualdad de género. Análisis de las acciones especificas de protección y de publicidad, Madrid, Dirección General de la Seguridad Social. Dirección http://www.seg-social.es/prdi00/groups/ public/documents/binario/097545.pdf. Web visitada el 5 de febrero de 2011. 
que su aplicación no está exenta de dificultades y de críticas sobre su contribución a reforzar este ro ${ }^{38}$. Finalmente, la Ley $2 / 2007$, conocida como ley de igualdad, asienta las bases del permiso de paternidad ${ }^{39}$ que, aunque insuficiente, es una medida encaminada a facilitar la corresponsabilidad.

Igualmente, el plan de igualdad en vigor ${ }^{40}$ revisa el concepto de conciliación y lo sustituye por el de corresponsabilidad. Retoma, así, uno de los significados de este término, recogido hace más de treinta años por la comunidad internacional. Este giro (de la conciliación a la corresponsabilidad) manifiesta la necesidad de aplicar la perspectiva de género a la propuesta de medidas y/o acciones.

En este sentido, se destacan cuatro ideas clave estrechamente relacionadas con el objetivo de fomentar la sensibilización, tratadas en la IV Conferencia de la Mujer de la ONU: la visibilidad, la paridad, el empoderamiento (o capacitación) y el mainstreaimg (o transversalidad, enfoque de género a todos los temas y políticas). Estas claves están estrechamente relacionadas con estrategias políticas para alcanzar la igualdad de oportunidades entre mujeres y hombres. Las tres primeras fueron garantes de la aplicación del enfoque de género a la comunicación mediática en otro estudio $^{41}$ y han sido operacionalizadas y utilizadas para la medición de los sesgos de género en la Ley General de Publicidad a partir de las modificaciones acometidas al amparo de la ley integral sobre violencia de género ${ }^{42}$.

Por todo lo mencionado es pertinente tratar de cuantificar las acciones realizadas por las administraciones regionales y locales en todo este periodo (1999-2007), desde el año que se aprueba la ley de conciliación hasta la aprobación de la ley de igualdad, como primera aproximación al estudio del esfuerzo de los gobiernos en materia de sensibilización en igualdad de género. Este trabajo se basa en la consideración de las acciones de comunicación y de formación promovidas por las instituciones públicas como necesarias para fomentar la sensibilización en el reparto equilibrado de responsabilidades familiares y laborales y, por tanto, como una de las vías para obtener mayores grados de igualdad. El artículo muestra el primer estudio que se ha llevado a cabo en España que cuantifica y analiza este tipo de acciones promovidas por la administración regional y local. Los resultados son una primera aproximación a la evaluación de estas políticas.

38 GARCíA, M. M., y LA PARRA, D. (2007): «La investigación sobre cuidados informales en salud desde una perspectiva de género», en Borrell, C. y ARTAZCOZ, L. (ed.), $5^{\circ}$ Monografía Sociedad Española de Epidemiología. Investigación en género y salud, Barcelona, Sociedad Española de Epidemiología, 97-118.

39 EsPaña. JEFATURA DEL ESTADO (2007): Ley Orgánica de para la igualdad efectiva de mujeres y hombres, (Ley 3/2007), BOE 71 (2007/03/23), 12611-12645.

40 Ministerio DE IGUALDAD (2008), op. cit.

41 Ruiz Cantero, M. T.; Martín, M.; La Parra, D.; Vives, C.; Albaladejo, M. et. al. (2004): «El enfoque de género en las noticias de salud», Gaceta Sanitaria, 18, Madrid, Elservier, SESPAS, 64-74.

42 Martín Llaguno, M.; Navarro Beltra, M. (2011): «Publicidad y leyes de violencia de género. Estudio empírico en España y Argentina», Revista Latina de Comunicación Social, La Laguna, Universidad de la Laguna (en prensa). 
El principal objetivo es analizar con enfoque de género (visibilidad, paridad, empoderamiento y mainstreaimg) las acciones de comunicación y formación vinculadas a temas de corresponsabilidad, como indicadores del esfuerzo orientado a promover la igualdad de género, por parte de seis gobiernos regionales, así como de sus capitales. Los objetivos concretos son, en primer lugar, registrar las acciones de comunicación y formación realizadas en todo el periodo. En segundo lugar, describir dichas acciones para valorar su contribución a la visibilización del problema de la corresponsabilidad, a la paridad, empoderamiento y mainstreaimg. Por último, cuantificar el esfuerzo realizado para promover la corresponsabilidad a través de acciones de formación y comunicación (incluida la publicidad a través de medios convencionales) por parte de los gobiernos de las seis regiones seleccionadas.

\section{Método}

El diseño del estudio es exploratorio con predominio del análisis documental y descriptivo (tabla I). El análisis se centra en las acciones de comunicación y formación vinculadas a temas de igualdad de género realizadas por parte de seis comunidades autónomas, así como de sus capitales. A modo de ejemplo, entre estas acciones estarían incluidas tanto la publicidad en medios convencionales (campañas en televisión, radio, cine, medio impreso, exterior e Internet) como en no convencionales (i.e. calendarios, guías, trípticos) de acuerdo con la clasificación que hace de los medios Infoadex ${ }^{43}$. También se analizan los cursos, las exposiciones, los ciclos o las conferencias.

Tabla I. Ficha Técnica

Diseño: Estudio exploratorio con predominio del análisis documental y descriptivo.

Periodo de observación: 1999-2007

Universo:

1. Acciones relacionadas con la corresponsabilidad, realizadas por las administraciones de Andalucía, Cataluña, Comunidad Valenciana, Galicia, Madrid y País Vasco, y por sus capitales. El tamaño del universo se desconoce.

2. Publicidad convencional realizada en las anteriores comunidades y ciudades. El tamaño del universo según Infoadex es de 136 anuncios (87 campañas).

Recogida de datos:

1. Control por declaración

2. Infoadex (hasta 2006)

Tamaño de la muestra recogida en este estudio:

1. En total se registraron 8.684 documentos. El $66 \%$ son objeto de estudio.

2. 136 anuncios de publicidad convencional (87 campañas)

Trabajo de campo: de febrero a julio 2008

Fuente: Elaboración propia.

43 SÁnchez ReVIlla, M. A. (2010): Resumen. Estudio Infoadex de la inversión de la comunicación publicitaria 2010, Madrid, Infoadex S.A., 11. Dirección http://www.infoadex.es/RESUMEN\%202010.pdf. Web visitada el 5 de febrero de 2011. 
El análisis se centra en el gobierno de Andalucía, en Cataluña, la Comunidad Valenciana, Galicia, Madrid y el País Vasco, así como en sus respectivas capitales: Sevilla, Barcelona, Valencia, La Coruña, Madrid y Bilbao. Las cinco primeras regiones lideran el ranking, entre las 17 comunidades autónomas que constituyen España, en cantidad de población. El País Vasco, aunque se ubica en el séptimo lugar, posee una densidad de población que la sitúa en segundo lugar (tras la Comunidad de Madrid) de entre las comunidades de la península. Asimismo fue seleccionado para completar las regiones que tienen lenguas distintas al español, además de una historia económica destacada; características que pudieran repercutir en su identidad cultural.

La información se recoge de cuatro tipos de instituciones: 1) Ayuntamientos de las capitales de las seis regiones, como fuente estrictamente local, 2) Diputaciones de las capitales, como fuente provincial, 3) Direcciones Generales de la Mujer o instituciones análogas como fuentes de información a nivel regional y, por último, 4) otras concejalías vinculadas al tema de la corresponsabilidad, regionales.

En primer lugar, se realiza un exploratorio de cada una de estas entidades, a través de su página Web oficial, con el objetivo de identificar las áreas específicas de trabajo, relacionadas con la igualdad de oportunidades, y fundamentalmente con la corresponsabilidad. En segundo lugar, se procede a contactar telefónicamente y se les envia por escrito una explicación detallada de la información solicitada, que consistía en: 1) memorias de las actividades realizadas con enfoque de género y 2) publicidad a través de medios no convencionales. El periodo de observación comprende desde 1999 a 2007 (ambos incluidos).

En consecuencia, esta investigación utiliza, entre otros procedimientos, el control por declaración. Se recurre a este método en los estudios que, como éste, precisan realizar un registro de las unidades de análisis. En el terreno de la publicidad, este tipo de control es frecuente en el recuento de las inversiones publicitarias de algunos medios. Una vez recopilado este material, se procedió a la depuración de la información obtenida y a su registro.

Tras lo expuesto, se incluyen todos aquellos documentos que puedan ser clasificados en los temas de interés para este estudio. Los temas y subtemas se extraen del Plan Estratégico de Igualdad de Oportunidades y se recogen en la ficha (tabla II). Con el ánimo de no perder información, no solo se incorpora el eje de corresponsabilidad del Plan vigente, sino también otros aspectos que, de acuerdo con la literatura especializada y de las actuaciones del propio Plan, pueden explicar la relación de la vida familiar, personal y laboral aunque estén insertos en otro eje de actuación. Se excluyen como objeto de estudio las actividades realizadas en materia de prevención y ayuda a la violencia machista.

Para el registro de las acciones se construye un protocolo de recogida de información que contiene la principales variables descriptivas (tabla II) que dan respuesta a la interpretación de los resultados de acuerdo con los cuatro conceptos 
mencionados: 1) Visibilidad, observada a través de la frecuencia (cantidad) de iniciativas y del tema; 2) Paridad o participación equilibrada, observada principalmente a través del público objetivo al que se dirige la iniciativa; 3) Empoderamiento o capacitación ${ }^{44}$, medido a través del tipo de acción que se realiza (predominantemente formativa, informativa o conmemorativa) y 4) Mainstreaming, observado a través de la permanencia en el tiempo de la iniciativa (o de la inversión) y la complementariedad con otras acciones que refuercen el mensaje.

Las variables fueron codificadas mediante el programa estadístico SPSS-15.

Para comparar entre regiones, se construyen dos indicadores que pretenden cuantificar el esfuerzo de las administraciones y que utilizan el tamaño de hábitat de las áreas y del público al que se destina la mayor parte de las acciones: el número de acciones realizadas por 100.000 habitantes y el número de acciones realizadas por cada 100.000 mujeres de 15 a 64 años.

Tabla II. Ficha de recogida de información

\begin{tabular}{|l|l|}
\hline 0) Identificador & P2. Subtema (multirrespuesta, hasta 3) \\
\hline P1. Tema (multirresp., hasta 3) & 1. Día Internacional de la Mujer \\
\hline 1.Igualdad de oportunidades & 2. Participación social y política de \\
la mujer & $\begin{array}{l}\text { 2. Asociacionismo. 3. Participación en puestos de } \\
\text { representación y decisión política. 4. Participación en } \\
\text { puestos directivos. 5. Redes de mujeres }\end{array}$ \\
\hline 3.Políticas públicas & $\begin{array}{l}\text { 6. Políticas de igualdad. 7. Transversalidad. } \\
\text { 8. Presupuestos públicos }\end{array}$ \\
\hline 4.Igualdad y calidad de ocupación & $\begin{array}{l}\text { 9. Barreras. 10. Formación ocupacional. 11. Implan- } \\
\text { tación plan de igualdad en empresas. 12. Prevención } \\
\text { del acoso }\end{array}$ \\
\hline 5.Corresponsabilidad & $\begin{array}{l}\text { 13. Servicios de atención y cuidado. 14. Estrategias } \\
\text { de conciliación. 15. Buenas prácticas en empresas. } \\
\text { 16. Ley de conciliación. 17. División sexual del tra- } \\
\text { bajo. 18. Valor del cuidado. 19. Nuevas tecnologías y } \\
\text { servicios }\end{array}$ \\
\hline 20. Dependencia. 21. Monoparentalidad \\
\hline 6.Familia & $\begin{array}{l}\text { 22. Historia de las mujeres científicas. 23. Mujeres } \\
\text { científicas. 24. Teorías feministas }\end{array}$ \\
\hline 7.Conocimiento & $\begin{array}{l}\text { 25. Lenguaje sexista. 26. Representación sexual en } \\
\text { contextos de producción. 27. Enfoque de género en } \\
\text { los medios }\end{array}$ \\
\hline 8.Comunicación & 28. Asesoramiento. 29.Formación operadores juríd. \\
\hline 9.Tutela del derecho a la igualdad & 31. ¿Cuál? \\
\hline 10.Urbanismo y género & (Nombre de la comunidad) \\
\hline 11.Otros -> &
\end{tabular}
hombres y mujeres, Bruselas, Dirección General de Empleo, Relaciones Laborales y Asuntos Sociales. 


\begin{tabular}{|c|c|}
\hline P4. Ciudad & (Nombre de la capital) \\
\hline P5. Institución pública & $\begin{array}{l}\text { 1. Ayuntamiento. 2. Diputación. 3. Direc. gnral. de } \\
\text { la mujer. 4. Otra concejalía -> ¿Cuál? }\end{array}$ \\
\hline $\begin{array}{l}\text { P6. Organismos colaboradores } \\
\text { (multirrespuesta) }\end{array}$ & $\begin{array}{l}\text { 1.Sindicatos. 2. Fondo Social Europeo. 3.Ministerios. } \\
\text { 4.Diputaciones. 5.Ayuntamientos. 6.Gobierno Re- } \\
\text { gional. 7. Otro }->\text { ¿Cuál? }\end{array}$ \\
\hline P7. Tipo de documentación & $\begin{array}{l}\text { (predominantemente formativa) } \\
\text { 1. Exposición, Ciclo. 2. Curso, Taller, Seminario, } \\
\text { Mesa de trabajo. 3. Jornada, Congresos, Encuentros. } \\
\text { 4. Conferencia. 5. Foro. 6. Becas. } \\
\text { (predominantemente informativa) } \\
\text { 7. Publicidad o soporte publicitario } \\
\text { 8.Otros. -> ¿Cuál? }\end{array}$ \\
\hline $\begin{array}{l}\text { P8. Tipo de acción conmemorativa } \\
\text { (celebrar fechas destacadas. Ej. Día } \\
\text { Inter. Mujer) }\end{array}$ & $\begin{array}{l}\text { 1.Sí } \\
\text { 2. No }\end{array}$ \\
\hline $\begin{array}{l}\text { P9. Fecha de inicio acción } \\
\text { P10. Fecha fin acción }\end{array}$ & $\begin{array}{l}\text { 1. Día. 2. Mes. 3. Año. } \\
\text { 1. Día. 2. Mes. 3. Año. }\end{array}$ \\
\hline \multirow{2}{*}{$\begin{array}{l}\text { Tipo de cur- } \\
\text { so/taller/seminario/mesa }\end{array}$} & P11. 1. Permanente -dos años-. 2. Puntual. \\
\hline & $\begin{array}{l}\text { P12. 1. Dependiente de un proyecto. - }>\text { ¿Cuál? } \\
\text { 2. No dependiente. }\end{array}$ \\
\hline $\begin{array}{l}\text { P13. No de horas del curso... } \\
\text { P14. No de plazas del curso... }\end{array}$ & $\begin{array}{l}\left.\text { ( }{ }^{\circ} \text { de horas }\right) \\
\left(\mathrm{n}^{\mathrm{o}} \text { de plazas }\right)\end{array}$ \\
\hline \multirow[t]{2}{*}{$\begin{array}{l}\text { Tipo de jorna- } \\
\text { da/congreso/encuentro... }\end{array}$} & $\begin{array}{l}\text { P15. 1. Permanente- }>\left(n^{0} \text { de edición } \_\right) \text {. } \\
\text { 2. Puntual. }\end{array}$ \\
\hline & $\begin{array}{l}\text { P16. 1. Dependiente de un proyecto. -> ¿Cuál? } \\
\text { 2. No dependiente. }\end{array}$ \\
\hline P17. Horas de las jornadas/con... & $\left(\mathrm{n}^{\mathrm{o}}\right.$ de horas $)$ \\
\hline P18. Publicidad no convencional & 1. Sí. 2. No \\
\hline P19. Soporte publicitario (I) & $\begin{array}{l}\text { 1. Mass media. 2. Póster. 3. Tríptico. 4. Calendario. } \\
\text { 5. Otro -> ¿Cuál? }\end{array}$ \\
\hline P20. Soporte publicitario (II) & $\begin{array}{l}\text { 1. Forma parte de una campaña. 2. Forma parte de un } \\
\text { curso/jornada... -> (código del evento __ } \\
\text { 3. Ninguna de las anteriores }\end{array}$ \\
\hline $\begin{array}{l}\text { P21. Target (multirrespuesta, hasta } \\
\text { 3) }\end{array}$ & $\begin{array}{l}\text { 1. Población general. 2. Hombres. 3. Mujeres. } 4 . \\
\text { Otros -> ¿Cuál? }\end{array}$ \\
\hline
\end{tabular}

Fuente: Elaboración propia.

La información sobre publicidad a través de medios convencionales fue proporcionada por Infoadex. Esta entidad se encarga del control de dicha publicidad emitida a través de los grandes medios publicitarios (televisión, radio, cine, medio impreso, exterior e Internet). Se trata del estudio tipo ADEX por excelencia en España. De estas campañas se conoce el tema, el anunciante, el medio, la fecha y la 
inversión real estimada ${ }^{45}$. Se utiliza la inversión como medida de esfuerzo ${ }^{46}$ de las campañas de igualdad de género y corresponsabilidad.

Para extraer estos datos, se tuvo que hacer una agrupación previa. Para ello, se atendió a lo que Infoadex codifica como «modelo», por el cual se consigue una brevísima descripción relacionada con el objeto de la campaña. Se agruparon todas las campañas en cuya descripción aparece el género, la mujer, la igualdad y a la corresponsabilidad. Las de violencia fueron eliminadas.

En este caso se hace un análisis de la inversión por años, por meses y medios, para conocer si obedecen a una estrategia de medios puntual o sostenida en el tiempo entre 1999 y 2006 (últimos datos disponibles en la base manejada). Igualmente se recoge el carácter informativo o persuasivo de las campañas analizadas ${ }^{47}$ atendiendo a la descripción de las mismas. Estos análisis permiten interpretar los resultados en su contribución a la visibilidad, paridad, empoderamiento y mainstreaming.

\section{Resultados}

\subsection{Recuento de las acciones de comunicación, formación y publicidad conven- cional}

En total se registraron 8.684 documentos de las administraciones regionales y locales, entre los cuales se encuentran tanto acciones de formación y comunicación como recursos de diferente naturaleza (informativos, económicos, infraestructuras). El 66\% son documentos objeto de estudio ( $\mathrm{N}=5.697$ ) (tabla III). De estos, el 79\% responden a acciones de carácter predominantemente formativo. La publicidad a través de medios no convencionales representa un $6 \%$.

La mayor parte de las acciones objeto de estudio han sido llevadas a cabo por las entidades regionales representantes de la igualdad de mujeres y hombres y/o concejalías, en contraste con los Ayuntamientos y Diputaciones.

La mitad de las acciones tienen vinculados organismos colaboradores, en concreto 2.604 registros. En estos casos predominan como colaboradores las instituciones públicas y sindicatos. Entre ellos resalta el mecenazgo de corporaciones locales (1142 iniciativas), del Fondo Social Europeo (944 iniciativas), del Gobierno de Galicia (la Xunta -89 iniciativas y Servicio Galego de Igualdade- 59 iniciativas), sindicatos (62 iniciativas) y del Ministerio de Trabajo y Asuntos Sociales (49

\footnotetext{
45 INFOADEX (2009): Libro Técnico, Madrid, Infoadex.

46 GonzÁlez Lobo, Ma A.; CArrero López, E. (2003): Manual de Planificación de Medios, Madrid, ESIC.

47 Kotler, P. (1986): Mercadotecnia, México, Prentice-Hall.
} 
iniciativas). El resto de organismos registrados (hasta 167) tienen asociadas menos de 30 iniciativas.

En cuanto a la publicidad a través de los mass media, se han identificado 136 anuncios de igualdad relacionados con mujer, género o corresponsabilidad que forman parte de 87 campañas cuyos anunciantes son algunas de las administraciones objeto de estudio entre 1999 y 2006.

Tabla III. Porcentaje de la documentación objeto de estudio sobre el total de documentación registrada que cumplía con los criterios de inclusión

\begin{tabular}{|l|r|r|r|}
\hline & Frecuencia & $\%$ & $\%$ válido \\
\hline 1. Cursos/talleres/Seminarios & 4485 & $51,6 \%$ & $78,7 \%$ \\
\hline $\begin{array}{l}\text { 2. Exposiciones/Ciclos/Jornadas/ } \\
\text { Congresos/Encuentros/Conferencias }\end{array}$ & 743 & $8,5 \%$ & $13 \%$ \\
\hline 3. Foros & 21 & $0,2 \%$ & $0,4 \%$ \\
\hline 4. Publicidad medios no convencionales & 354 & $4 \%$ & $6,2 \%$ \\
\hline 5. Recursos informativos (ex. $\mathrm{n}^{\circ}$ teléfono) & 94 & $1 \%$ & $1,7 \%$ \\
\hline Total análisis & 5697 & $\mathbf{6 5 , 6 \%}$ & $100 \%$ \\
\hline Perdidos & 2987 & $34,4 \%$ & \\
\hline Total registros & 8684 & $100 \%$ & \\
\hline
\end{tabular}

Fuente: Elaboración propia.

\subsection{Análisis de las acciones: conceptos clave}

\subsubsection{Tipo de acciones llevadas a cabo y permanencia de la iniciativa}

Entre todas las acciones destacan las acciones de carácter formativo. La mayor parte de los cursos (88\%) son permanentes (presentes en dos o más años) y dependientes de otro proyecto. Es decir, no constituyen iniciativas aisladas sino integradas en una propuesta de mayor envergadura, como puede ser la campaña en torno al Día Internacional de la Mujer, o insertas en proyectos subvencionados por el Fondo Social Europeo. Suelen durar menos de 20 horas y van dirigidas a un grupo reducido.

Los eventos tales como ciclos, exposiciones, encuentros, jornadas o conferencias, que tienen la posibilidad de dirigirse a un público más amplio que los cursos, representan el $13 \%$ de todas las iniciativas consideradas. Hay menos eventos de este tipo que tienen continuidad (el 45\%) y menos dependientes de otros proyectos (el 36\%). Las jornadas, encuentros y congresos duran, casi todos ellos, menos de 20 horas.

La publicidad a través de medios no convencionales constituye aproximadamente el 6\% (tabla III). Las 354 piezas registradas son fundamentalmente pósters, trípticos, calendarios, pegatinas o agendas entre otros obsequios, cuya capacidad para llegar a públicos amplios es más limitada que en el caso de la publicidad a 
través de los medios de comunicación. De tales piezas, 41 hacen referencia a un mismo evento o campaña.

En la recogida de información se incluyeron también recursos. Algunos de ellos, los que serán objeto de análisis, son acciones relacionadas con una acción de comunicación (ej. un número de teléfono). Otros no son estrictamente acciones de formación o de comunicación aunque están estrechamente vinculados con el objeto de investigación (ej. los centros de día o ayudas económicas asociadas).

\subsubsection{Temas sobre los que versan las acciones y público objetivo}

Las iniciativas versan fundamentalmente sobre «igualdad y calidad de la ocupación». De lejos le siguen los temas sobre «corresponsabilidad», «conocimiento»y «políticas públicas». Entre los subtemas destacan la «formación ocupacional», «barreras contra el empleo femenino» $\mathrm{y}$ «conciliación de la vida familiar y profesional».

Se han recogido hasta 64 targets distintos. Las acciones registradas van dirigidas fundamentalmente a mujeres entre 15 y 64 años. Un porcentaje bastante menor se dirige a la población general y a hombres de 15 a 64 años. Otro tipo de público es el interno de las instituciones. En este caso, resaltan los Ayuntamientos y las Diputaciones.

\subsection{Análisis de las acciones: cuantificación del esfuerzo}

Las áreas que registran en total (gobierno regional, provincial y local) un mayor número de acciones son Madrid y Andalucía (tabla IV). El 92\% de las acciones promovidas por Madrid y el $86 \%$ de las iniciativas de Andalucía son cursos. Se observa cómo Cataluña, Galicia y el País Vasco apuestan por las Exposiciones/Ciclos/Jornadas/Congresos/Encuentros o Conferencias.

Tabla IV. Iniciativas por tipo de acción

\begin{tabular}{|l|r|r|r|r|r|r|r|r|}
\cline { 2 - 9 } \multicolumn{1}{c|}{} & 1 & 2 & 3 & 4 & 5 & N fila (\%) & \% col. & N regional \\
\hline Andalucía & $86 \%$ & $5 \%$ & $0 \%$ & $7 \%$ & $2 \%$ & $1920(100 \%)$ & $33,7 \%$ & 1850 \\
\hline Cataluña & $38 \%$ & $51 \%$ & $1 \%$ & $8 \%$ & $3 \%$ & 535 & $9,4 \%$ & 364 \\
\hline C. Valenc. & $67 \%$ & $18 \%$ & $0 \%$ & $10 \%$ & $4 \%$ & 263 & $4,6 \%$ & 136 \\
\hline Galicia & $27 \%$ & $49 \%$ & $1 \%$ & $22 \%$ & $1 \%$ & 252 & $4,4 \%$ & 198 \\
\hline Madrid & $92 \%$ & $4 \%$ & $0 \%$ & $3 \%$ & $1 \%$ & 2517 & $44,2 \%$ & 2416 \\
\hline País Vasco & $32 \%$ & $47 \%$ & $1 \%$ & $17 \%$ & $3 \%$ & 210 & $3,7 \%$ & 194 \\
\hline N columna & 5485 & 743 & 21 & 354 & 94 & 5697 & $100 \%$ & \\
\cline { 1 - 8 } & & & & & &
\end{tabular}

Fuente: Elaboración propia. (Nota: Códigos en la tabla III.)

Así, destacan claramente Madrid y Andalucía en el número de iniciativas no vinculadas a la publicidad por medios convencionales. Esta tendencia persiste in- 
cluso cuando se analizan las acciones teniendo en cuenta el volumen de población de cada región, sea para el total o únicamente para las mujeres activas (tabla $\mathrm{V}$ ).

Según los indicadores creados para observar el esfuerzo sometiéndolo al número de habitantes (columna D) y al número de mujeres entre 15 y 64 años (columna E) se observa que las regiones, en su conjunto, se ubican de igual forma en ambos casos. Destaca Madrid y le siguen Andalucía, País Vasco, Galicia, Cataluña y, finalmente, Valencia. El dato mayor son 123 acciones por cada 100.000 mujeres de entre 15 y 64 años en todo el periodo ( 9 años), lo que correspondería a 1 acción por 7.142 mujeres en un año en el mejor de los casos.

Tabla V. Acciones por cien mil habitantes y acciones por cien mil mujeres de 15 a 64 años. Acciones totales de la Comunidad Autónoma + capital (ciudad y provincia)

\begin{tabular}{|l|r|r|r|r|r|}
\hline & \multicolumn{1}{|c|}{$\begin{array}{c}\text { (A) } \\
\text { Acciones } \\
(1999-2007)\end{array}$} & $\begin{array}{c}\text { (B) } \\
\text { Población media } \\
(1999-2007)\end{array}$ & $\begin{array}{c}\text { Población media } \\
\text { mujeres 15-64 } \\
(1999-2007)\end{array}$ & $\begin{array}{c}\text { (D) } \\
=100.000(\mathrm{~A}) \\
\text { (B) }\end{array}$ & $\begin{array}{c}\text { (E) } \\
=100.000(\mathrm{~A}) \\
/(\mathrm{C})\end{array}$ \\
\hline Andalucía & 1920 & 7.634 .096 & 2.597 .311 & 25,2 & 73,9 \\
\hline Cataluña & 535 & 6.688 .357 & 2.277 .819 & 8,0 & 23,5 \\
\hline C.Valencia. & 262 & 4.457 .233 & 1.522 .402 & 5,9 & 17,2 \\
\hline Galicia & 252 & 2.748 .541 & 930.174 & 9,2 & 27,1 \\
\hline Madrid & 2517 & 5.647 .567 & 2.043 .847 & 44,6 & 123,2 \\
\hline País Vasco & 210 & 2.115 .185 & 741.306 & 9,9 & 28,3 \\
\hline
\end{tabular}

Fuente: Elaboración propia. Los datos de población se extraen del padrón en el INE.

\subsection{Publicidad e inversión en los medios de comunicación de masas}

El 55\% de las campañas objeto de estudio son de carácter informativo (ej. sobre el Día Internacional de la Mujer, el Plan de Igualdad de Oportunidades o la Dirección General de la Mujer). Se distribuyen de la siguiente forma: 55 anuncios (30 campañas) en Madrid; 26 anuncios (20) en Cataluña; 20 (13) en el País Vasco; 18 (12) en Galicia; 9 (7) en Andalucía y 8 (5) en Valencia.

En términos de inversión se observa que la mayor parte de las regiones apuestan por la prensa como principal medio publicitario, seguida de la televisión (tabla VI). La única excepción es el País Vasco, que prefiere utilizar la televisión. 
Tabla VI. Media de inversión real estimada periodo 1999-2006

\begin{tabular}{|l|r|r|r|r|r|r|r|}
\hline & $\begin{array}{c}\text { Inversión } \\
\text { media \% col. }\end{array}$ & $\begin{array}{c}\text { Exterior } \\
\text { \% Fila }\end{array}$ & $\begin{array}{c}\text { Prensa } \\
\text { \% Fila }\end{array}$ & $\begin{array}{c}\text { Cine } \\
\text { \% Fila }\end{array}$ & $\begin{array}{c}\text { Radio } \\
\text { \% Fila }\end{array}$ & $\begin{array}{c}\text { TV } \\
\text { \% Fila }\end{array}$ & $\begin{array}{c}\text { Internet } \\
\text { \% Fila }\end{array}$ \\
\hline Andalucía & $2,13 \%$ & 0,00 & 70,49 & 0,00 & 0,00 & 29,51 & 0,00 \\
\hline Cataluña & $19,62 \%$ & 0,79 & 59,97 & 0,00 & 0,00 & 38,77 & 0,47 \\
\hline C. Valencia & $3,28 \%$ & 16,97 & 68,02 & 0,00 & 0,00 & 15,00 & 0,00 \\
\hline Galicia & $6,08 \%$ & 3,96 & 49,36 & 4,97 & 0,00 & 41,71 & 0,00 \\
\hline Madrid & $56,17 \%$ & 5,20 & 35,83 & 0,00 & 22,58 & 33,53 & 2,85 \\
\hline P. Vasco & $12,73 \%$ & 25,03 & 21,54 & 5,30 & 0,00 & 48,13 & 0,00 \\
\hline N (\% col.) & $503.797,01(100 \%)$ & & &
\end{tabular}

Fuente: Elaboración propia a partir de los datos de Infoadex.

Sobre la inversión media total del sector de campañas de interés público, la inversión media efectuada por estas comunidades autónomas en publicidad para todo el periodo supone un $0,31 \%$ (tabla VII) sobre el sector (campañas de interés público). La cuota media de todos los Ministerios es del $45 \%$ y solo las campañas de tráfico representan el 14,7\% (1999-2006). A nivel nacional, la inversión efectuada por los Ministerios en campañas de igualdad supone el $0,7 \%$ del total del sector.

Tabla VII. Comparación de las cuotas de inversión medias (1999-2006) sobre sector

\begin{tabular}{|l|r|l|r|l|r|}
\hline \multicolumn{3}{|l|}{ Cuota de inversión de las Regiones } & \multicolumn{2}{l|}{ Cuota de inversión Ministerios } \\
\hline Andalucía & $0,007 \%$ & Galicia & $0,020 \%$ & Cuota Ministerios* & $45,16 \%$ \\
\hline Cataluña & $0,063 \%$ & Madrid & $0,181 \%$ & Cuota campañas género** & $0,69 \%$ \\
\hline Valencia & $0,011 \%$ & P. Vasco & $0,041 \%$ & Cuota campañas tráfico** & $14,68 \%$ \\
\hline \multicolumn{4}{|c|}{ Cuota regiones sobre el total: $0,31 \%$} & &
\end{tabular}

Fuente: Elaboración propia con datos proporcionados por Infoadex.

Por años, la inversión es irregular en el periodo considerado. Los años 2005 y 2006 concentran la mayor parte de la inversión efectuada en todo el periodo (31 y $21 \%$ respectivamente). Tras estos dos años, destaca el 2002 (17\%). El peor año es 1999 , con un $2 \%$ de inversión. Todos los demás están en torno al $7 \%$.

En cuanto a la distribución de la inversión por regiones (gráfico I) se observan tres tendencias distintas que no agrupan las áreas en términos de distribución geográfica. La inversión de Galicia y Madrid crece de forma destacada los últimos dos años. En contraste, se aprecia cómo la inversión de la Comunidad Valenciana y el País Vasco decrece a lo largo del periodo. La inversión de Cataluña y Andalucía es cíclica, con diferencias destacadas entre años (de hasta un 30\% de diferencia interanual). 
Gráfico I. Cuota de la inversión real estimada sobre el total de inversión de las Comunidades Autónomas por años

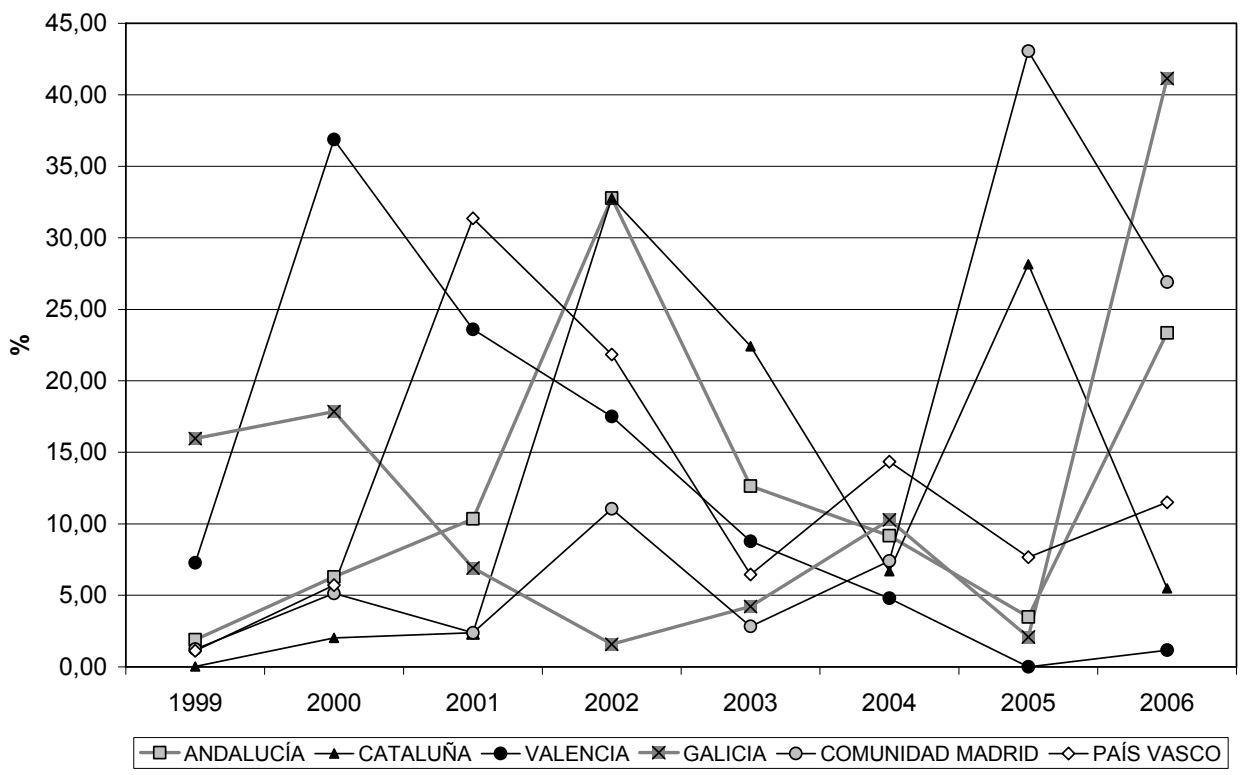

Fuente: Elaboración propia a partir de los datos de Infoadex.

\section{Conclusiones y discusión}

Esta investigación facilita información que no ha sido recogida con anterioridad. En términos absolutos, como respuesta al primer objetivo, se ha registrado un número de iniciativas considerablemente elevado. Una explicación posible residiría en el apoyo -especialmente de índole económica- recibido, en muchos casos, de la mano del Fondo Social Europeo. Ahora bien, si se observan las iniciativas más sobresalientes (predominan los cursos de formación ocupacional), los temas y el carácter de las campañas convencionales, se debe considerar que los recursos económicos públicos manejados no han sido utilizados para promover estrategias de sensibilización, como fueron definidas a la luz de los documentos internacionales. Se ha dado un espacio menor a otros temas relacionados con la igualdad de género, la corresponsabilidad, la difusión del conocimiento, el desarrollo de políticas públicas y otros propios de la comunicación (i.e. representaciones de género, estereotipos). Igualmente, como apunta la descripción que las identifica en Infoadex, muchas de las campañas son puntuales (ej. Día Internacional de la Mujer) e informativas.

El predominio de la formación ocupacional dentro de las agendas regionales y locales vinculadas a la igualdad de género lleva a considerar que el enfoque desde 
el que se plantean iniciativas para el fomento de la igualdad de género es fundamentalmente laboral, con la intención tanto de fomentar la incorporación de las mujeres al mercado de trabajo como de enseñar el desarrollo de destrezas que permitan la promoción en su empresa. Se trataría de iniciativas vinculadas a políticas de empleo cuya relación con las políticas de igualdad es, sobre todo, el público al que se dirige: es decir, las mujeres. De acuerdo con el segundo objetivo, esta cuestión evidencia la necesidad de mayores grados de mainstreaming. Además, el claro predominio de las mujeres como grupo objetivo, sea en los cursos o en otras acciones, revela posibles carencias en términos de paridad, pues corre el riesgo de transmitir la idea de que el problema de la falta de igualdad es un asunto exclusivamente de ellas. Este hecho no se daría si las acciones de formación y comunicación se insertaran en una estrategia política que hubiera asimilado la perspectiva de género con la pretensión de sensibilizar en corresponsabilidad.

Pese a todo ello, se advierte la existencia de una voluntad de sensibilización en lo que concierne a la visibilidad del problema, aunque ciertamente es más un efecto colateral en el caso de las acciones de formación y comunicación. Las exposiciones, la publicidad en medios no convencionales y la publicidad a través de los medios de comunicación facilitan más este objetivo que los cursos formativos. El elevado número -en total y en términos absolutos- de tales iniciativas lleva a considerar que se han visibilizado los problemas relacionados con las desigualdades de género, aunque hayan sido dirigidas a grupos reducidos. Además, la cuota de inversión publicitaria del conjunto de las áreas geográficas de estudio frente a la cuota nacional apoya también esta afirmación.

A favor de los cursos formativos se debe decir que son iniciativas dirigidas a conferir algún tipo de capacidad o recurso al grupo de mujeres, por lo que, desde esta perspectiva, contribuyen al empoderamiento, al igual que todas las acciones informativas.

Finalmente, en cuanto al tercer objetivo, el número de iniciativas por habitante es pequeño. Esta cifra queda muy lejos de la cantidad de población total e, incluso, del grupo de mujeres de 15 a 64 años, y esto es así pese a que se han sumado las acciones efectuadas en 9 años. Si a este dato se le une el hecho de que la mayor parte de las iniciativas van dirigidas a grupos poblaciones pequeños, se puede concluir que son escasas en términos de cobertura.

Algunas acciones tienen mucho más potencial para generar cobertura, como es el caso de las campañas publicitarias a través de los medios de comunicación de masas. Sin embargo, el predominio del medio impreso, especialmente de los diarios, hace que estas campañas se dirijan a un tipo de público más segmentado, con un nivel sociocultural medio y medio-alto. Además, se comprende que la presencia de estas campañas en televisión puede ser menos relevante de lo que el dato de inversión muestra, ya que el umbral de inversión para que el mensaje no pase desapercibido en este medio es elevado, debido a la saturación publicitaria. En consecuencia, el esfuerzo, medido en 
términos de inversión publicitaria, realizado por las comunidades analizadas tampoco se puede considerar relevante en términos absolutos.

Con todo, en términos comparativos, la inversión publicitaria regional y local supone casi la mitad de la inversión nacional en campañas de igualdad, pese a que se han estudiado 6 de las 17 regiones posibles. Estos datos reflejan, por tanto, un esfuerzo de los gobiernos regionales y de sus capitales en materia de publicidad convencional, sobre todo por parte de Madrid.

El impacto real de todas las acciones (formación, comunicación y publicidad a través de medios de comunicación) en términos de sensibilización se entremezcla con otro tipo de intervenciones, incluso, con situaciones estructurales que condicionan los cambios. No obstante, se debe destacar, como otra fortaleza de la investigación, que la información recogida, aunque no permite averiguar los efectos, sin embargo permite dilucidar si existe una estrategia política y de comunicación que apueste por la sensibilización de manera sostenida en el tiempo. En este sentido, se puede afirmar que las acciones o iniciativas de comunicación y formación promovidas por las administraciones consideradas no parecen estar integradas en una estrategia a medio-largo plazo. Muchos cursos tienen continuidad en tanto que están vinculados al Día Internacional de la Mujer. Sin embargo, el hecho de que este Día tenga una frecuencia anual no hace de estas actividades una estrategia sostenida. Más bien se trata de acciones puntuales que se repiten al menos durante dos años. Incluso las campañas publicitarias a través de los mass media, que precisan mayores inversiones y en las que el planteamiento estratégico debería ser el punto de partida, no son mantenidas en el medio plazo.

En consecuencia, desde la teoría feminista de la comunicación, las intervenciones serían insuficientes para producir un efecto, pues, como ya se dijo, de existir, debería ser el resultado de una acción global y sostenida. Además, desde la perspectiva el marketing social y por todo lo expuesto, también se advertirían carencias en la estrategia, tanto a corto como a medio y largo plazo, si el objetivo a considerar es la sensibilización en corresponsabilidad. Queda por observar, como implicación de este estudio, otros factores explicativos que no han sido analizados y pueden responder a un planteamiento estratégico de otra índole. Para ello parece oportuno analizar longitudinalmente todos los datos con el propósito de encontrar posibles coincidencias con intereses o situaciones que dieran una respuesta a las diferencias detectadas entre comunidades (elecciones, promoción de leyes, cierre de presupuestos, agenda...).

En definitiva, de todas las iniciativas analizadas, las campañas publicitarias contribuyen a la visibilización del problema y las acciones llevadas a cabo, fundamentalmente, contribuyen al empoderamiento, pero no se trabaja el objetivo de la paridad. Los resultados apuntan a una falta de estrategia política a medio y largo plazo en materia de sensibilización en todas las regiones analizadas y a la necesidad de mayores grados de mainstreaming. Si bien los datos proporcionan una idea del esfuerzo realizado por las instituciones locales (provinciales) y regionales, también 
reflejan la necesidad de seguir insistiendo en la elaboración de estrategias políticas orientadas a la sensibilización acordes con el enfoque de género, el cual da sentido al proyecto de igualdad de oportunidades entre mujeres y hombres. Un proyecto que, desde hace más de medio siglo, está consensuado internacionalmente.

\section{Referencias bibliográficas}

Brown, M. E. (1994): «Feminist Culturalist televisión Criticism - Culture, Theory and Practice» en Mary Ellen Brown (ed.): Television and Women's Culture. The Politics of the Popular, London, Sage Publications, 11-22.

CANel, M. J. (2007): Comunicación de las Instituciones Públicas, Madrid, Tecnos.

CIS (2004): Opiniones y actitudes sobre la familia, $n^{\circ} 2578$, Madrid, CIS.

- (2008): Barómetro de Junio, $n^{\circ}$ 2766, Madrid, CIS.

Dow, B. J.; ConDIT, C. (2005): «The State of the Art in Feminist Scholarship in Communication», Journal of Communication, 3, Washington: ICA / Blackwell Publishing, 448478.

DunCAN, S. (2002): «Policy discourses on "Reconciling Work and Life" in the EU», Social Policy \& Society, 1, Cambridge, Cambridge University Press, 305-314.

DURÁN, R.; GINER, J. I. (1999): «Vivir la discapacidad (campaña de sensibilización y cambio de actitudes sobre la discapacidad en edad escolar)», Revista de Servicios Sociales y Política Social, 48, Madrid, Consejo General de Colegios de Diplomados en Trabajo Social, 75-84.

España. JefatuRa Del Estado (1999): Ley de la Conciliación de la vida familiar y laboral (Ley 39/1999), BOE 266 (1999/11/06), 38934-38942.

- (2005): Ley de Publicidad y Comunicación Institucional (Ley 29/2005), BOE 312 (2005/12/30), 42902-42905.

- (2007): Ley Orgánica de para la igualdad efectiva de mujeres y hombres, (Ley 3/2007), BOE 71 (2007/03/23), 12611-12645.

EUROPA (1998): 100 palabras para la igualdad. Glosario de términos relativos a la igualdad entre hombres y mujeres, Bruselas, Dirección General de Empleo, Relaciones Laborales y Asuntos Sociales.

EuROPEAN COMMISSION (2000): Council Decision 2001/51/EC of 20 December 2000 establishing a Programme relating to the Community framework strategy on gender equality (2001-2005), Brussels, Directorate-General for Employment, Social Affairs and Equal Opportunities.

- (2006): Roadmap for equality between women and men 2006-2010, Communication from the Commission to the Council, the European Parliament, Brussels, European Economic and Social Committee and the Committee of the Regions.

García, M. (2001): Publicidad Institucional: el Estado anunciante, Málaga, Servicio de Publicaciones e Intercambio Científico de la Universidad de Málaga.

GARCÍA, M. M.; LA PARRA, D. (2007): «La investigación sobre cuidados informales en salud desde una perspectiva de género», en Borrell, C.; Artazcoz, L. (eds.), $5^{\circ}$ Monografia Sociedad Española de Epidemiología. Investigación en género y salud, Barcelona, Sociedad Española de Epidemiología. 
GonzÁlez Lobo, Ma A.; CARrero LóPez, E. (2003): Manual de Planificación de Medios, Madrid, ESIC.

INE (2009a): Mujeres y hombres en España 2009, Madrid, Instituto Nacional de Estadística.

- (2009b): Encuesta de Población Activa, ler trimestre de 2009, Madrid, Instituto Nacional de Estadística.

INFOADEX (2009): Libro Técnico, Madrid, Infoadex.

InSTITUTO DE LA MUJER (1999): Las Conferencias Mundiales de Naciones Unidas sobre las Mujeres, Madrid, Ministerio de Trabajo y Asuntos Sociales.

KLINTH, R. (2008): «The Best of Both Worlds? Fatherhood and Gender Equality in Swedish paternity Leave Campaigns, 1976-2006», Fathering, 1, New York, MetaPress, 2039.

Kotler, P. (1986): Mercadotecnia, México, Prentice-Hall.

Kotler, P.; LeE, N. (2006): Marketing in the Public Sector: A Roadmap for Improved Performance, Philadelphia, Wharton School Publishing.

Kotler, P.; RoBerto, E. (1989): Social Marketing: Strategies for Changing Public Behavior, New York, Free Press.

Martín Llaguno, M.; Navarro Beltra, M. (2011): «Publicidad y leyes de violencia de género. Estudio empírico en España y Argentina», Revista Latina de Comunicación Social, La Laguna, Universidad de la Laguna (en prensa).

MartíneZ, E.; VizCaíno-LaORGa, J.; GaViLÁN, R. (2008): «Government Advertising: an integrative element. The legal framework in Spain», Revista Latina de Comunicación Social, 63, La Laguna, Laboratorio de Tecnologías de la Información y Nuevos Análisis de Comunicación Social.

Mattelart, A.; Mattelart, M. (1998): Theories of Communication: A Short Introduction, Inbunden, Sage Publications.

Mattelart, A.; NeveU, E. (2003): Introducción a los estudios culturales, Barcelona, Paidós.

Ministerio DE IGUALDAD (2008): Plan Estratégico de Igualdad de Oportunidades entre Mujeres y Hombres (2008-2011), Madrid, Ministerio de Igualdad.

- (2008): Plan Estratégico de Igualdad de Oportunidades entre Mujeres y Hombres (2008-2011), Madrid, Ministerio de Igualdad.

PAPí-GÁlveZ, N. (2008): El género entre bastidores. El caso de las periodistas de la Comunidad Valenciana, Alicante, Universidad de Alicante.

- (dir.) (2004): Evaluación del impacto de la conciliación entre la vida familiar y profesional para la igualdad de género. Análisis de las acciones específicas de protección y de publicidad, Madrid, Dirección General de la S. S. Dirección http://www.segsocial.es/prdi00/groups/public/documents/binario/097545.pdf. Web visitada el 5 de febrero de 2011.

Papí-GÁlveZ, N.; Orbea Mira, J. (2011): Comunicación institucional eficaz en igualdad de género. Sensibilizar en corresponsabilidad. Claves y recomendaciones, Madrid, Asociación de la Comunicación Publicitaria.

Rice, R. E.; ATKIN, C.K. (eds.) (2001): Public Communication Campaigns, California, Sage Publication. 
Ruiz Cantero, M. T.; Martín, M. ; La Parra, D.; Vives, C.; Albaladejo, M. et. al. (2004): «El enfoque de género en las noticias de salud», Gaceta Sanitaria, 18, Madrid, Elservier, SESPAS, 64-74.

SÁnchez ReVILLA, M. A. (2010): Resumen. Estudio Infoadex de la inversión de la comunicación publicitaria 2010, Madrid, Infoadex S.A. Dirección http://www.infoadex.es/RESUMEN\%202010.pdf. Web visitada el 1 de febrero de 2011.

Santesmases Mestre, M. (1999): Marketing. Conceptos y estrategias. 4a Edición. Madrid, Pirámide.

StRATIGAKI, M. (2004): «The cooptation of Gender Concepts in EU Policies: The Case of Reconciliation of Work and Family», Social Politics,1, Oxford, Oxford University Press, 30-56.

VAn Zoonen, L. (2004): Feminist Media Studies, London, SAGE Publications.

WolIN, L. (2003): «Gender Issues in Advertising- An Oversight Synthesis of Research: 1970-2002», Journal of Advertising Research, 1, Cambridge, Cambridge University Press, 111-129.

Recibido: 30 de noviembre de 2010

Aceptado: 14 de marzo de 2011 